\title{
A New Methodology for Network Scale Simulation of Emerging Power Line Communication Standards
}

\author{
Stephen Robson, Abderrahmane Haddad, Member, IEEE, and Huw Griffiths, Member, IEEE
}

\begin{abstract}
This paper presents the development of a new methodology for the simulation of Power Line Communication (PLC) within the popular Electromagnetic Transients Programme-Alternative Transients Programme (EMTP-ATP). As a first application, the comparative performance of the emerging Orthogonal Frequency Division Multiplexing (OFDM) based Prime and G3-PLC narrowband standards is investigated. Models of both standards have been created within the EMTPATP and simulations performed using frequency dependent line models and realistic transformer models as terminations. It is observed that both standards are severely affected by the highly frequency selective nature of the MV power line channel and a more considered choice of OFDM parameters may be necessary for optimal performance on MV networks.
\end{abstract}

Index Terms-Narrowband PLC, Prime, G3-PLC, OFDM.

\section{INTRODUCTION}

$\mathbf{I}^{\mathrm{T}}$ $\mathrm{T}$ is widely agreed that to implement future smart grids, there needs to be greater network visibility. This requires more communication. However, on rural and semi-rural networks, the expenditure of a dedicated communication infrastructure is often not justifiable, and it is generally considered that inexpensive alternatives should be developed to satisfy the future requirements [1]. PLC has long been suggested as a technology to facilitate communication on grids. Recent examples include the use of PLC as a facilitator of an enhanced protection scheme on rural overhead networks [2], and a telemetry network using PLC based communication and the retrieval of synchrophasor information has also been examined as potential payload in a PLC based communication scheme. Arguably, recent PLC implementations over MV networks have been optimised for generic point to point use (IP based systems) rather than low latency multinode use [3][4]. Broadband over Power Line (BPL), for example, has been shown to be capable of providing high bandwidth links over large networks. However, the high frequencies demanded by BPL leads to high attenuation and the requirement for repeaters, adding an incremental cost to implementation, making it prohibitively expensive for smart grid implementations. More recently, Prime and G3-PLC have emerged as narrowband PLC standards, but their use and published field trial results are predominantly for "electrically short" low voltage networks. This paper examines the performance of these standards in large, rural networks that approach the condition of being "electrically

S. Robson and A. Haddad are with the Advanced High Voltage Engineering Research Centre (AHIVE), Cardiff University, UK. H. Griffiths is with Petroleum Institute, Abu Dhabi, UAE. e-mail: robsons1@ cardiff.ac.uk

Manuscript received April 19, 2005; revised January 11, 2007. long" and explores the possibility of further modifications to improve the robustness of narrowband PLC on such networks. The target application for such a modified communication protocol is for the retrieval of telemetry information (e.g. synchronised voltage and current or travelling wave fault recorder information) on rural $11 \mathrm{kV}$ networks, where the potential benefits may currently be outweighed by the cost of existing systems.

The method used in this paper relies on a direct implementation of an OFDM modulator in the EMTP-ATP. The flexible Fortran based MODELS language is used to model a generic OFDM modulator with configurable parameters, allowing several proposed schemes to be tested, including the emerging PRIME and G3-PLC standards [5][6]. The advantage of this approach over traditional channel model techniques is the ability to determine quickly the performance of the communication scheme between various points on the network. It has been shown in previous studies, and in this investigation, that the performance of PLC over large networks can vary substantially depending on where the transmitter and receiver are located, and on the presence of strong resonance effects at localised frequencies. Therefore, it is proposed that network-wide studies should be used to evaluate PLC technologies. A further advantage of the presented method is its ability to assess performance during power system events such as switching surges, lightning strikes and fault conditions. Although it may be argued that these events are relatively rare, it is perhaps of increased importance that PLC performs robustly in these scenarios because the perceived value of telemetry information (i.e. voltage, current or transient values) is greater here than at times of normal operating conditions. The line models incorporated in the EMTP-ATP simulation use the frequency dependent J.Marti model, adopted extensively in transient analysis and the study of other high frequency phenomena.

\section{EXISTING PLC STANDARDS}

There are similarities but also some significant differences between the physical layers of the Prime and G3-PLC standards, a summary of which is shown in Table I. Both occupy the CENELEC A frequency band [7] (CENELEC norm EN. 50065-1). Prime is based on Differential Phase Shift Keying in frequency (DPSK- $f$ ), and as such, has chosen a relatively small subcarrier spacing. G3-PLC uses Differential Phase Shift Keying in time (DPSK-t), with a much larger subcarrier spacing. The symbol duration is larger in Prime partly due to a 
lower sampling frequency and partly due to a larger FFT size. The Cyclic Prefix (CP) is also significantly larger in Prime than in G3-PLC. The performance of Prime and G3-PLC will ultimately depend on several factors including the RMS Delay Spread (RMS-DS) of the channel, the coherence bandwidth, levels of Additive White Gaussian Noise (AWGN) and the frequency and severity of impulsive noise.

\section{Methodology}

This work uses a configurable simulation testbed for assessing the performance of various OFDM modulation scheme, as depicted in Fig. 1. The testbed makes use of both EMTPATP and Matlab to simulate a fully configurable OFDM communication system, with transmitter and receiver separated by a channel comprising frequency dependent line models. The transmitter is coded in the native models language within the EMTP-ATP and can easily be configured to a custom $\mathrm{CP}$, sampling rate, FFT size and subcarrier modulation. The transmitter is also capable of generating a synchronisation symbol appended to the start of a transmission. The OFDM output signal is injected into the network via a mutually coupled RL element with mutual inductance chosen to match commercial inductive couplers operating in the CENELEC A band. The network is made up of frequency dependent J.Marti line models based on a typical wood pole $11 \mathrm{kV}$ line, common in the rural distribution network of the UK. The injected signal propagates through the network, and the time domain signals at various positions are exported to the receiver model in Matlab for demodulation. The receiver comprises an optional synchronisation and FFT process for demodulation of the OFDM signal. After demapping, a bit error calculation is made by comparing the received bits with the bits that were sent from the transmitter. Given that field trials of narrowband PLC have shown that the position of the receiver, relative to the transmitter, has a large effect on performance [8], the ability to

TABLE I

OVERVIEW OF THE PRIME AND G3-PLC STANDARDS [5][6]

\begin{tabular}{|c|c|c|}
\hline & Prime & G3-PLC \\
\hline Frequency & $42-89 \mathrm{kHz}$ & $35-91 \mathrm{kHz}$ \\
\hline Sampling Frequency, $f_{s}$ & $250 \mathrm{kHz}$ & $400 \mathrm{kHz}$ \\
\hline FFT Size & 512 & 256 \\
\hline Symbol Duration & $2048 \mu \mathrm{s}$ & $640 \mu \mathrm{s}$ \\
\hline Cyclic Prefix (samples) & 48 & 30 \\
\hline Cyclic Prefix (duration) & $192 \mu \mathrm{s}$ & $75 \mu \mathrm{s}$ \\
\hline Subcarrier spacing, $\Delta f$ & $488.28125 \mathrm{~Hz}$ & $1.5625 \mathrm{kHz}$ \\
\hline Used subcarriers & 97 & 36 \\
\hline Windowing & No & Yes \\
\hline \multirow[t]{2}{*}{ Modulation } & \multicolumn{2}{|c|}{ DBPSK, DQPSK, D8PSK } \\
\hline & In Frequency & In Time \\
\hline Data Rate (maximum) & $128.6 \mathrm{kbps}$ & $33.4 \mathrm{kbps}$ \\
\hline Interleaving & Per Symbol & Per Packet \\
\hline
\end{tabular}

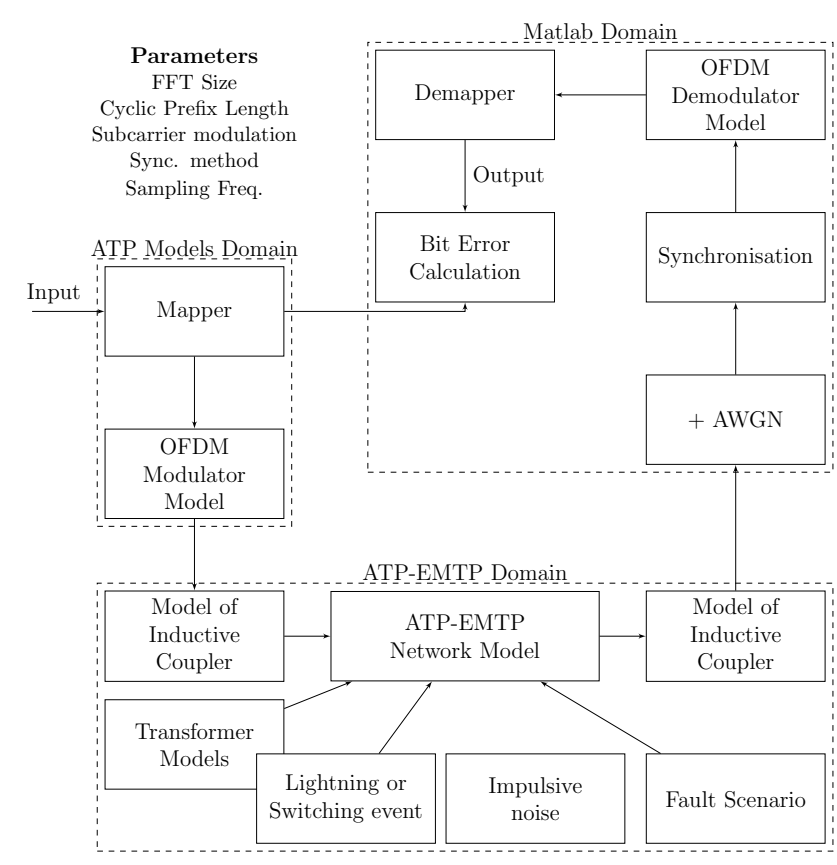

Fig. 1. Block diagram of the simulation setup. The configurable parameters are FFT Size, CP length, subcarrier modulation, synchronisation method and sampling frequency. The parameters adopted for Prime and G3-PLC are summarised in Table 1

consider several receiver positions simultaneously could be an important advantage of the EMTP-ATP method as opposed to using generic channel models. Furthermore, the EMTP-ATP offers possibilities in imposing realistic network events like switching, lightning strikes and the presence of a noise source at specific locations on the network. The performance of the communication scheme under such conditions is of increased importance because it is during these events, and immediately after, that information from remote parts of the network may be the most valuable.

\section{A. Line Models}

To model the frequency dependence of overhead lines, the J.Marti frequency-dependent line model is chosen [10]. This model has been used extensively in the literature for modelling transients in the tens and hundreds of $\mathrm{kHz}$ range [11], and is therefore deemed appropriate for studies of PLC communication in the CENELEC A band (35-90 kHz).

The test network chosen for this study is based on the $11 \mathrm{kV}$ rural model within the United Kingdom Generic Distribution Network (UKGDS) [9]. This network is representative of a typical rural MV network with overhead lines. Table II summarises the main characteristics of the line and Fig. 2 shows the network under study.

\section{B. Transformer Models and Line Terminations}

On a rural/semi rural MV network, there will typically be a single HV/MV transformer at the primary substation feeding a radial, usually branched network with dispersed 

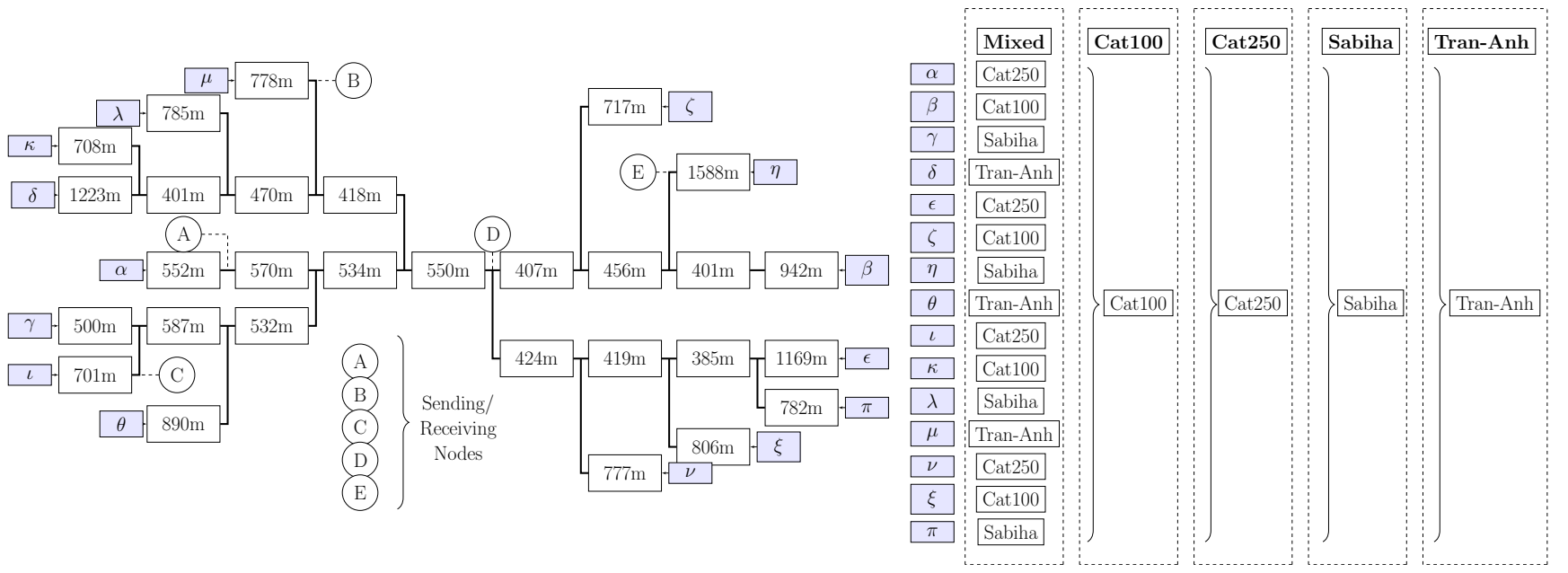

Fig. 2. The Test Network based on the semi-rural United Kingdom Generic Distribution System [9]. Transformer terminations are at the end of each branch (labelled $\alpha-\pi$, based on lumped element models of the Cataliotti $100 \mathrm{kVA}$ and $250 \mathrm{kVA}$ models, the Sabiha model and the Tran-Anh model. A mixed assumption, with terminations randomly assigned, is also shown.

TABLE II

CHARACTERISTICS OF THE OHTL UNDER TEST

\begin{tabular}{lc}
\hline \multicolumn{2}{c}{$11 \mathrm{kV}$ Wood Pole Overhead Line } \\
\hline Height, $h$ & $9 \mathrm{~m}$ \\
Height at mid-span, $h_{\text {mid }}$ & $7.5 \mathrm{~m}$ \\
Conductor separation, $d$ & $1.5 \mathrm{~m}$ \\
Conductor name & Dingo \\
Conductor core area & $158.7 \mathrm{~mm}^{2}$ \\
Conductor resistivity & $0.1814 \Omega / \mathrm{km} \mathrm{DC}$ \\
\hline
\end{tabular}

MV/LV transformers (both single phase and three phase). It is reasonable to assume that in most cases, each branch will terminate with an $\mathrm{MV} / \mathrm{LV}$ transformer. In some cases, however, the branch may have an open circuit termination because it adjoins a normally open point or it is feeding single phase loads. As the main termination of the branches on the network, it is therefore extremely important to model accurately the high frequency behaviour of the transformer in PLC studies.

In this work, four types of high frequency transformer models have been implemented within the EMTP-ATP; Tran-Anh's model of a $160 \mathrm{kVA}$ distibution transformer [12], Sabiha's model of a $100 \mathrm{kVA}$ transformer [13] and Cataliotti's models of $100 \mathrm{kVA}$ and $250 \mathrm{kVA}$ transformers [14]. These lumped element models have been fitted against empirical data from real distribution transformers across the range of frequencies occupied by Prime and G3-PLC, as detailed in Table III. Here, the input impedance is defined as the impedance presented to a travelling wave by the transformer. Plots of the magnitude and phase of the transformer input impedance according to the models are shown in Fig. 3.

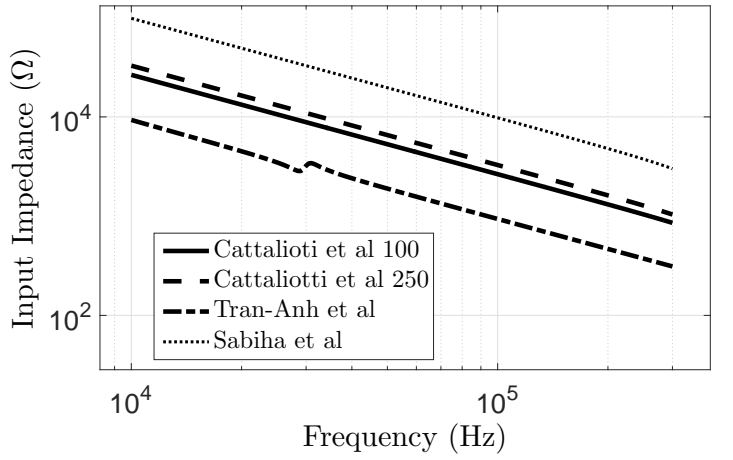

(a) Magnitude of the transformer input impedance

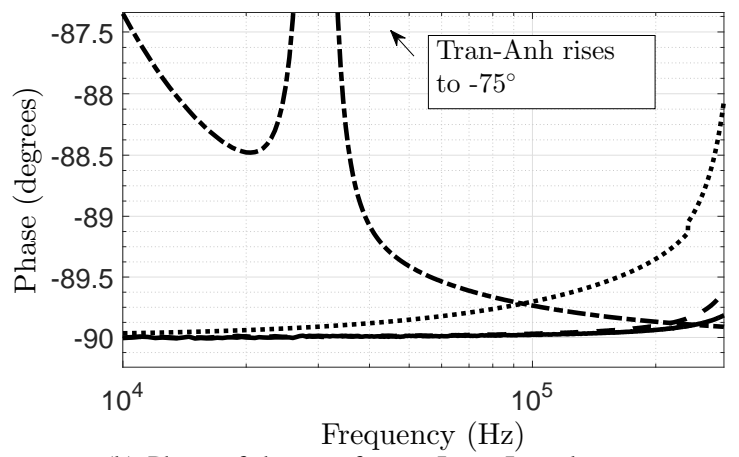

(b) Phase of the transformer Input Impedance

Fig. 3. Magnitude and Phase of the transformer input impedance of the four lumped element models, between $10 \mathrm{kHz}$ and $300 \mathrm{kHz}$. The resonance in the Tran-Anh model increases the phase to $-75^{\circ}$ at approximately $30 \mathrm{kHz}$.

TABLE III

OVERVIEW OF THE TRANSFORMER MODELS

\begin{tabular}{lll}
\hline Model Name & Rated Power & Rated Voltage \\
\hline Tran-Anh et al [12] & $160 \mathrm{kVA}$ & 20kV/400V \\
Cataliotti et al 100 [14] & $100 \mathrm{kVA}$ & $\mathrm{MV} / \mathrm{LV}$ \\
Cataliotti et al 250 [14] & $250 \mathrm{kVA}$ & $\mathrm{MV} / \mathrm{LV}$ \\
Sabiha et al [13] & $100 \mathrm{kVA}$ & $21 \mathrm{kV} / 420 \mathrm{~V}$ \\
\hline
\end{tabular}




\section{Coupling}

Coupling to the network is required to inject, and extract the PLC signal to and from the network. In this work, inductive coupling is implemented using the mutually coupled RL element of the EMTP, with the overhead line acting as primary, and the secondary is where the signal from the OFDM MODEM is applied. A mutual inductance of 1000 $\mathrm{nH}$ is assumed as a reasonable approximation to commercially available inductive couplers operating in the CENELEC range. Throughout this study, coupling to aerial mode 2 , i.e. the mode involving both outer conductors of the three phase line, is assumed. This choice is driven by the large attenuation of the ground mode, which is high enough to neglect in practical PLC studies involving a lossy ground [15].

\section{Channel Characteristics}

\section{A. Channel Impulse Response and Delay Spread on the MV Channel}

There have been numerous studies examining the channel characteristics of the power line channel. Some have concentrated on obtaining empirical measurements of the RMSDS and the impulse response, mainly on LV channels and at frequencies beyond $30 \mathrm{MHz}$ [16][17]. Others aim to construct a useful channel model based on these empirical results [18][19][20]. However, it may be generally assumed that prior to the deployment of a communication device on an MV power line, detailed knowledge of the channel response between any two arbitrary points is not available, as is demonstrated by the lack of published results in the literature.

Frequency selective fading is caused by multipath interference and results in an irreducible error floor at signal bandwidths that exceed the coherence bandwidth. The problem of frequency selective fading is emphasised in the power line channel because the transmitted signal is bound by the power line structure, ensuring that there will be at least as many time-delayed reflections reaching the receiver as there are branches and, typically, more as the signal continues to propagate throughout the network. The power of the timedelayed version of the transmitted signal is limited by the attenuation, giving rise to a diminishing received power. This metric is usually referred to as the RMS-DS of the channel. To avoid this impairment, the symbol duration can be set to greater than the RMS-DS of the channel. Therefore, the RMS-DS sets a limit on the signalling rate in single carrier modulation schemes and has prompted the use of multi-carrier modulation schemes such as OFDM.

The impulse response of a channel is defined in terms of the input, $u(t)$, output, $y(t)$ and the delay spread function, $h(t, \tau)$.

$$
y(t)=u(t) \star h(t, \tau)=\int_{-\infty}^{\infty} h(t, \tau) u(t-\tau) . d \tau
$$

The impulse response can also be used to calculate the RMS-DS. The first moment of the Power Delay Profile (PDP) is defined as:

$$
\bar{\tau}=\frac{\Sigma_{k} P\left(\tau_{k}\right) \tau_{k}}{\Sigma_{k} P\left(\tau_{k}\right)}
$$

Where $\bar{\tau}$ is the mean excess delay. The square root of the second central moment of the PDP is given by:

$$
\sigma_{\tau}=\sqrt{\frac{\Sigma_{k}\left[\tau_{k}-\bar{\tau}\right]^{2} P\left(\tau_{k}\right)}{\Sigma_{k} P\left(\tau_{k}\right)}}
$$

From the RMS-DS, one may also calculate the coherence bandwidth. Here, the definition shown below is used.

$$
B_{c}=\frac{1}{5 \sigma_{t}}
$$

Where $\sigma_{t}$ is the RMS delay spread and $B_{c}$ is the $50 \%$ coherence bandwidth. The RMS-DS is calculated for all possible channels involving nodes A to E (25 channels) for each transformer termination scenario (Tran-Anh, Cataliotti 250 kVA, 100 kVA, Sabiha and Mixed). i.e., where each branch is terminated according to Fig. 2. The simulations are repeated for additional transformers installed in the middle of each block (i.e, with reference to Fig. 2, in the middle of the $552 \mathrm{~m}$ block, the middle of the $570 \mathrm{~m}$ block etc). Two further cases are modelled, first, the special case of the line being terminated by an approximation to its characteristic impedance, in this case $300 \Omega$ for the aerial modes. Second, the case of the line being terminated by an open circuit. The resulting RMS-DS for each case is shown in Fig. 4.

For all four transformer termination assumptions, an RMSDS in the region of $200-900 \mu$ s is observed, with some extreme outliers surpassing $1 \mathrm{~ms}$. This is comparable to the RMSDS observed for the open circuit assumption, indicating that the modelled transformer terminations are behaving like open circuits. There is a small increase in RMS-DS observed when transformers are placed in the middle of each branch. The lowest RMS-DS is observed in the case of each branch terminated by an approximation to its characteristic impedance.

Importantly, the CP duration for Prime and G3-PLC (192 $\mu \mathrm{s}$ and $75 \mu \mathrm{s}$, respectively) are significantly shorter than the RMS-DS for almost all channels with a realistic transformer termination. Only for the unrealistic case of characteristic impedance terminations ( $\mathrm{Z}=300 \Omega$ case) are the durations of the delay spreads less than the CPs of Prime and G3-PLC. Fig. 5 shows the coherence bandwidth plotted as a function of sending/receiving node and termination assumption.

The impulse responses derived from EMTP-ATP simulations are also used to determine the magnitude and phase responses characterising each pair of nodes. Fig. 6(a) shows the magnitude responses for the Tran-Anh, Cataliotti 250 $\mathrm{kVA}$, the $\mathrm{Z}=300 \Omega$ (characteristic impedance) and open circuit assumptions (the Mixed, Cataliotti $100 \mathrm{kVA}$ and Sabiha assumptions exhibited similar characteristics to the Cataliotti 250 kVA and Tran-Anh cases, so are omitted from the figures). In all but the $\mathrm{Z}=300 \Omega$ case, a high degree of frequency selectivity is observed, with deep notches in the magnitude response in various regions. It is interesting to observe that the positioning of the notches are similar for the Tran-Anh, Cataliotti 250 $\mathrm{kVA}$ and Open circuit cases, suggesting that the geometry of the network (which is the same for all scenarios) is the main determinant. This result may be significant in the development of adaptive schemes which attempt to avoid regions with deep 


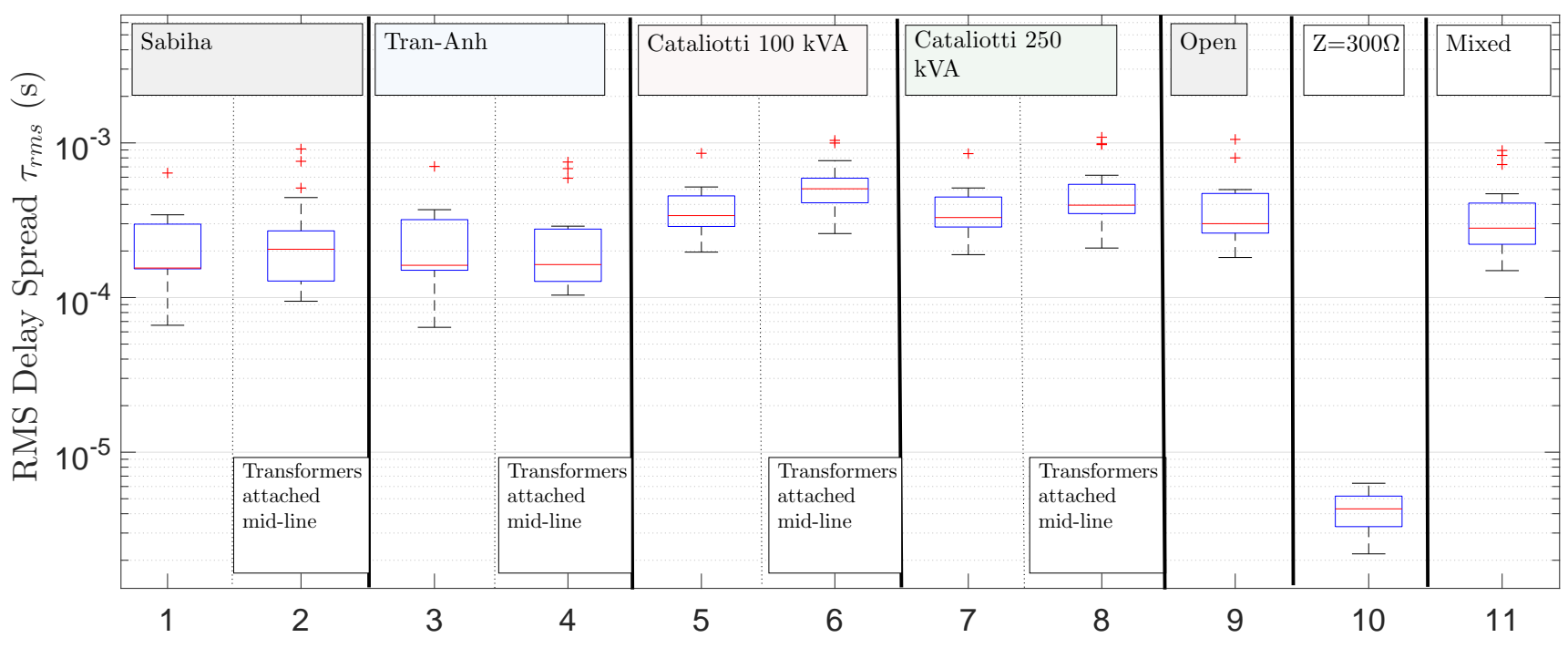

Fig. 4. Box-Whisker plot of the RMS Delay Spreads for different transformer terminations including an open circuit termination (i.e. no transformer) and a $300 \Omega$ impedance termination, chosen to be similar to the characteristic impedance of mode 2 of the distribution line.

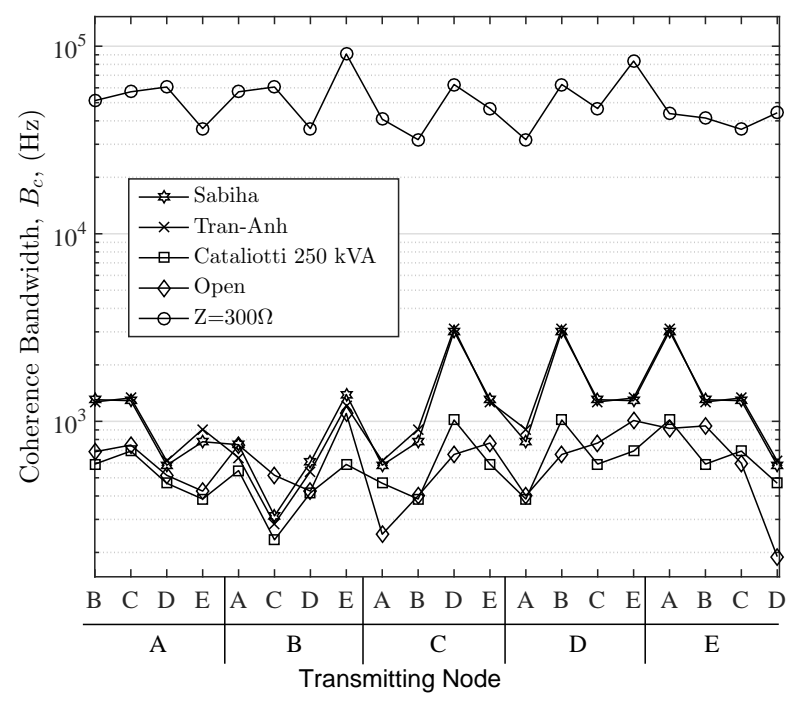

Fig. 5. 50\% Coherence Bandwidth between all node pairs on the example network, including the Sabiha, Tran-Anh, Cataliotti, open circuit and $Z=300 \Omega$ transformer termination assumptions.

notches or excessive phase shifts. Fig. 6(b) shows the phase responses for the selected channels. As would be expected, the positioning of phase non-linearities in frequency corresponds to the positioning of the notches in the magnitude response. The linearity of the phase response is of particular importance in Prime, which uses DPSK in frequency by encoding information in adjacent subcarriers. Such a scheme is prone to impairments because each subcarrier will be phase-rotated by differing amounts by the channel. From the phase response, the phase rotations between adjacent positions in the frequency domain can be calculated, as shown for a $600 \mathrm{~Hz}$ separation in Fig. 6(c). Any phase rotation beyond the decision threshold $\left(\frac{\pi}{2}, \frac{\pi}{4}\right.$ and $\frac{\pi}{8}$ for DBPSK, DQPSK and D8PSK, indicated by the horizontal dashed lines in Fig. 6(c)) will probably result in an error solely brought about by the channel itself. It is observed generally that the cases using a realistic transformer termination, and the open circuit assumption, exhibit more severe impairment than for communication between the same two nodes but with the $\mathrm{Z}=300 \Omega$ termination assumption. In all cases, the magnitude and frequency of the spikes decrease with increasing frequency, which would suggest that transmitting at higher frequencies would result in fewer errors of this kind, and therefore a lower irreducible error floor.

\section{Performance of OFDM Modulation Schemes on THE TEST NETWORK}

\section{A. OFDM}

A single OFDM symbol has a useful period $T$ preceded by a CP of length $T_{g}$. For Intersymbol Interference (ISI) free communication, the CP length, $T_{g}$, is longer than the channel impulse response. The OFDM time domain signal is defined as:

$$
v(t)=\sum_{k=-N}^{N} X_{k} e^{j 2 \pi k t / T},-T_{g} \leq t \leq T
$$

Where $X_{k}$ are the data symbols, $N$ is the number of subcarriers and $T$ is the OFDM symbol time. The frequencies of the complex exponentials are define as:

$$
f_{k}=\frac{k}{T},-N \leq k \leq N
$$

Prior to transmission, the signal is quadrature modulated and up-converted. The total useful period, $T$ increases as the number of sub-carriers, $k$ increases and is related by the sampling frequency, $f_{s}$.

$$
T=\frac{k}{f_{s}}
$$

It is commonly stated that for ISI free communication, the $\mathrm{CP}$ length should be greater than twice the RMS delay spread 


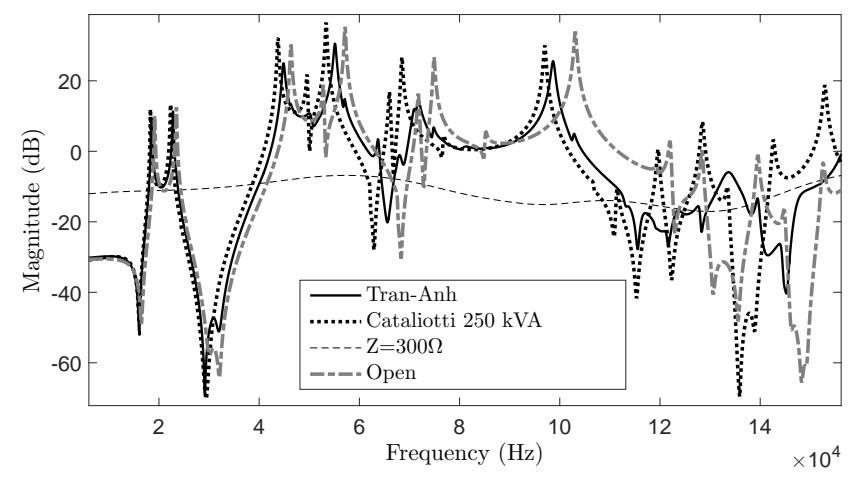

(a) Magnitude Response, A to B, various terminations

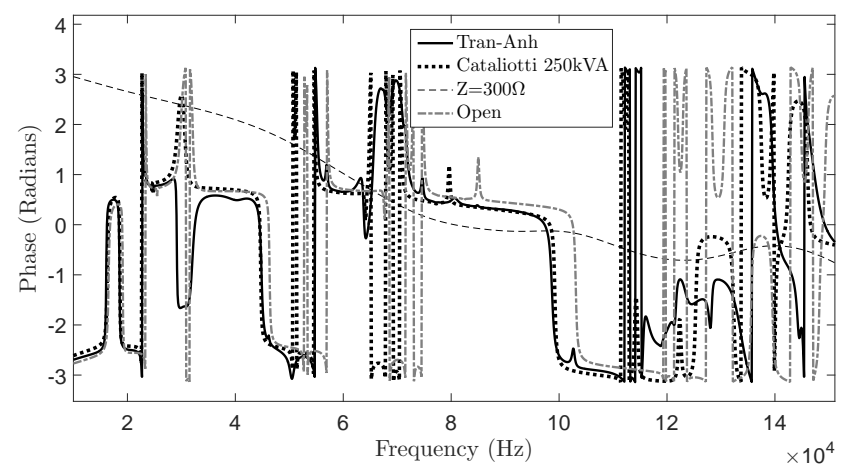

(b) Phase Response, A to B, various terminations

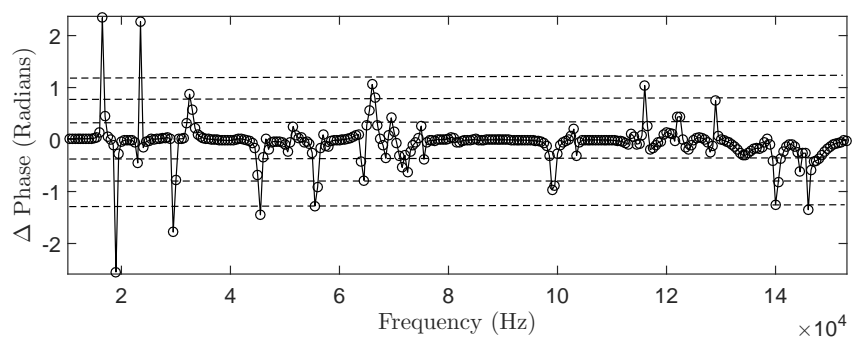

(c) $\Delta$ phase $(600 \mathrm{~Hz}$ intervals) for the Cat250 kVA termination.

Fig. 6. Magnitude, phase and $\Delta$ phase for the channel separating nodes A and $\mathrm{B}$.

of the channel. Furthermore, the subcarrier spacing should be less than the coherence bandwidth of the channel [21].

\section{B. Channel Performance of G3-PLC and PRIME on the UKGDS Test Network}

According to the methodology outlined earlier in section III, the physical layers of the Prime and G3-PLC standards are replicated in a simulation testbed involving EMTP-ATP and Matlab. The flexibility of the test setup allows for a large number of parameters to be analysed, including the effect of changing the $\mathrm{CP}$, the sampling frequency, subcarrier modulation and the termination assumption. The results are shown in the form of Box-Whisker plots, which show the statistical distribution of BER across all 25 possible channels in each scenario.

1) Raw BER Performance of Prime and G3-PLC as specified by the standards: Results 1 to 32 in Fig. 7 show the BER performance for Prime and G3-PLC for different termination scenarios and with different subcarrier modulation schemes. The $\mathrm{Z}=300 \Omega$ termination scenario (with all branches terminated with a resistor approximating the overhead line's characteristic impedance) is omitted from the figure because all BERs are zero.

It is observed that Prime performs slightly better than G3PLC for all subcarrier modulation schemes and termination assumptions. A possible explanation for these results is the relatively lower ISI experienced by Prime due to its larger $\mathrm{CP}$ than that used by G3-PLC. This advantage seems to outweigh the penalty paid by Prime for encoding in frequency rather than in time.

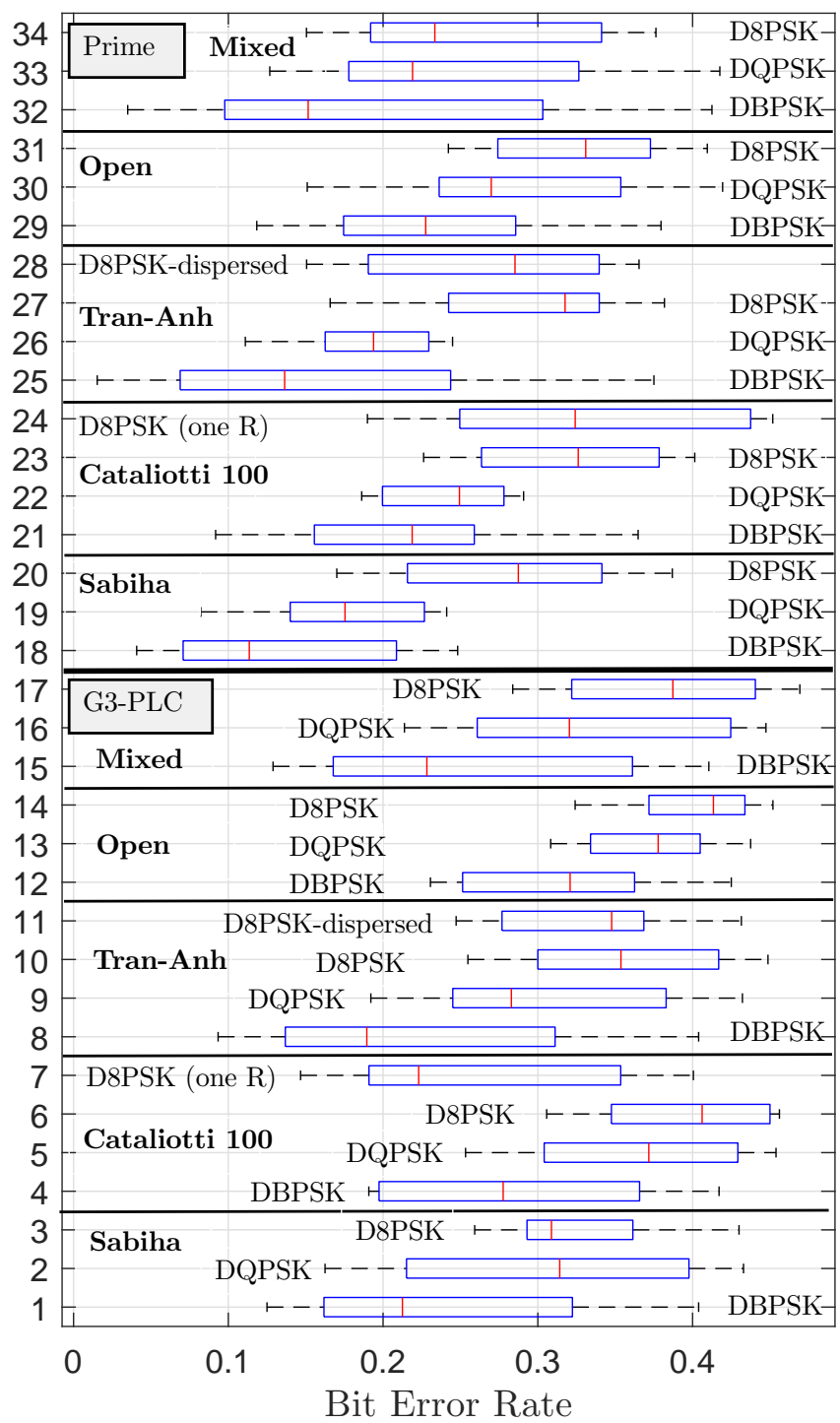

Fig. 7. Box-whisker plot showing the performance of Prime (top half) and G3-PLC (bottom half) for different transformer termination assumptions. The central line represents the median value, the edges of the box represents the $75^{t h}$ and $25^{t h}$ percentiles, the whiskers extend to the most extreme values not considered outliers and the outliers are plotted individually. The data used to produce each box and whisker are the range of BERs for communication between each node on the example network. In all cases, communication is noise-free. 
Overall, the BERs are lowest for the Sabiha and Tran-Anh transformer termination assumptions. Examination of Fig. 3 shows that both of these models are slightly less capacitive than the Cataliotti models across the CENELEC A frequencies. This gives a slightly closer match to the predominantly real characteristic impedance of the overhead line, therefore giving a possible explanation for the discrepancy in BERs. It is striking, however, that such small differences in the assumed transformer termination has resulted in relatively large differences in BER. Results 23 and 7 show the special case of terminating one of the branches (termination $\beta$, as shown in Fig. 2, for Prime and G3-PLC, respectively. It is interesting to note that for G3-PLC, there is a significant improvement in performance, though for Prime, a minimal change is observed.

2) Effect of changing the Sampling Frequency, $f_{s}$ : Fig. 8 shows the effect of decreasing the sampling rate to half of the original rate (for D8PSK and an assumed transformer termination of Cataliotti $100 \mathrm{kVA}$ ). For Prime, this would decrease $f_{s}$ to $125 \mathrm{kHz}$, and for G3-PLC, $f_{s}$ would decrease to $200 \mathrm{kHz}$. This change will have three main effects; a) the symbol duration would increase by a factor of $2, b$ ), the length of the CP would also increase by a factor of 2 , and c) the subcarrier spacing would be decreased by a factor of 2 . All three of these effects would be expected to increase the BER performance. The observed results show a considerable decrease in BER for Prime but a negligible effect for G3-PLC.

3) Effect of changing the CP: Fig. 9 shows the effect of changing the $\mathrm{CP}$ length with an assumed subcarrier modulation scheme of D8PSK and a termination assumption of Cataliotti $100 \mathrm{kVA}$. For Prime, a step change improvement is observed when the CP is increased from 144 samples $(576 \mu$ s) to 192 samples $(768 \mu \mathrm{s})$. There are further improvements in BER up to a $\mathrm{CP}$ of 600 samples, but no further gain beyond this. It is interesting to note that a $\mathrm{CP}$ of 192 samples (in the case of Prime) is approximately the mean RMS-DS for the Cataliotti 100 kVA termination assumption (as shown in Fig. 4).

For G3-PLC, a decrease in BER is observed at a CP of 300 samples $(750 \mu \mathrm{s})$, which again coincides with the mean RMS-DS of the channels between all combinations of nodes A to E. Doubling this CP to 600 samples leads to a significant improvement in BER, but there is only a small improvement in BER if the CP is further increased to 900 samples. It is noticeable that even with a CP of 900, the BER of G3-PLC has a similar performance to Prime with its nominal $\mathrm{CP}$ of

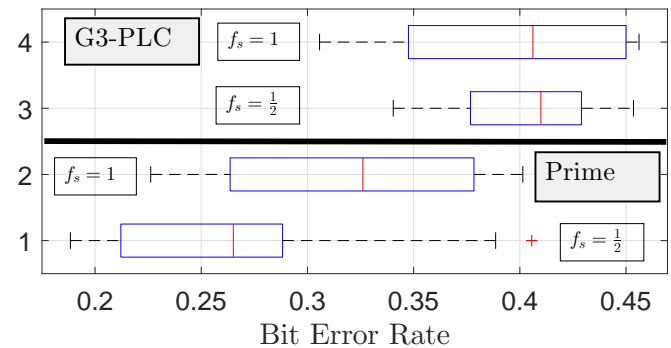

Fig. 8. Box-whisker plot showing the effect of decreasing the sampling frequency, $f_{s}$. The subcarrier modulation scheme is D8PSK and the transformer termination assumption is Cataliotti $100 \mathrm{kVA}$.

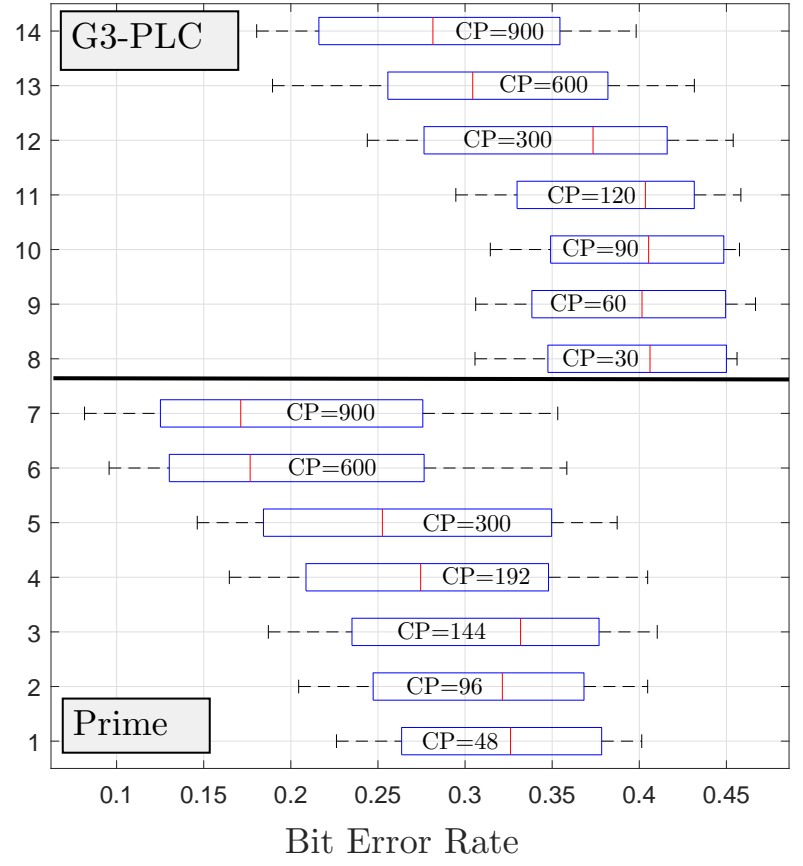

Fig. 9. Box-whisker plot showing the effect on BER of changing the number of samples in the CP. The modulation scheme is D8PSK and the termination is the Cataliotti $100 \mathrm{kVA}$ model in all cases.

just 48 samples $(192 \mu \mathrm{s})$.

4) Effect of changing the Carrier Frequency, $f_{c}$ : Fig. 10 shows the change in BER as the carrier frequency is increased (for the Cataliotti $100 \mathrm{kVA}$ and open circuit termination assumptions). For both Prime and G3-PLC, the BER gradually approaches zero as $f_{c}$ is increased. This would concur with the theoretical observation made in Section IV, that the channel response is more favourable at higher frequencies.

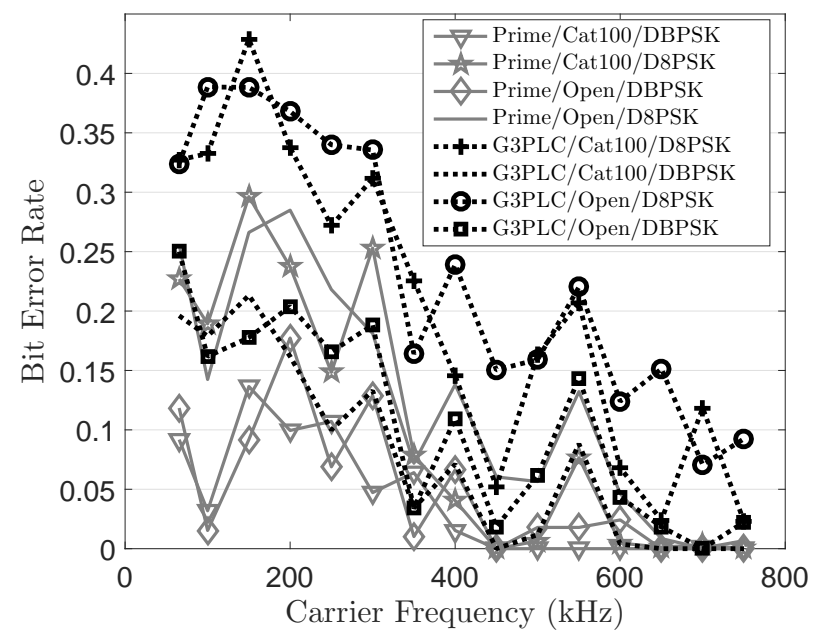

Fig. 10. BER as a function of carrier frequency for the Cataliotti and Open circuit termination assumptions. The BER performance for both DBPSK and D8PSK tends to zero as the carrier frequency is increased. 


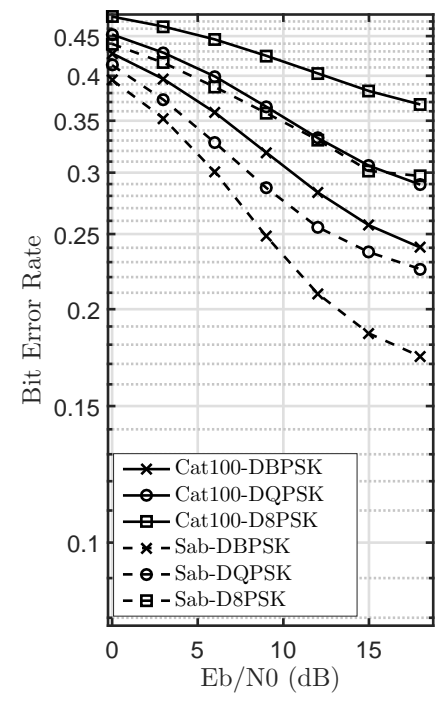

(a) G3-PLC

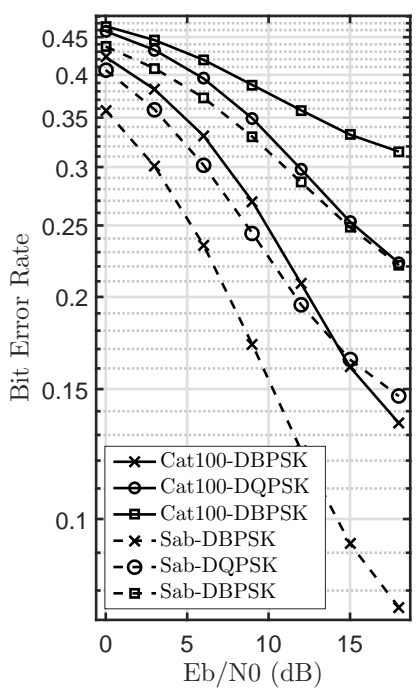

(b) Prime
Fig. 11. Bit Error Rate performance of Prime DBPSK and D8PSK for communication between node A and node D on the base network with Sabiha and Cataliotti $100 \mathrm{kVA}$ termination assumptions.

\section{Performance under the presence of Additive White Gaus- sian Noise (AWGN)}

Fig. 11 shows waterfall diagrams demonstrating the BER performance for communication between node A and D (chosen arbitrarily) under the presence of AWGN. Performance with the Sabiha assumption is noticeably better than the Cataliotti assumption for all subcarrier modulation schemes and all values of $E b / N 0$. It is also clear that Prime is generally better performing for all cases.

\section{BER by Subcarrier and the potential of adaptive schemes}

Fig. 12 shows the BER by subcarrier for noise-free communication between nodes $\mathrm{B}$ and $\mathrm{D}$ (arbitrarily chosen), and for 7 consecutive symbols in the case of Prime (Fig. 12(a)-12(c)), and 20 consecutive symbols in the case of G3-PLC (Fig. 12(d)12(f)). Three cases are shown for each standard; Open circuit transformer terminations, Mixed transformer terminations and Sabiha transformer terminations (chosen arbitrarily). It is noted that the errors are concentrated on groups of subcarriers, presumably due to the highly frequency selective nature of the channel. For example, in Prime, subcarriers 0 to 10,30 to 40 and 90 to 97 are severely affected, with BERs no better than 0.5 in these regions. In G3-PLC, a similar pattern is evident, with subcarriers 15 to 20 and 34 to 36 being severely affected. It is interesting to note that the severely affected regions in Prime and G3-PLC occupy the same frequency range. This result was observed for communication across all node combinations. The positive aspect of this result is the presence of large regions which are free from errors. This emphasises the importance of adaptive schemes which can dynamically allocate information to favourable subcarriers.

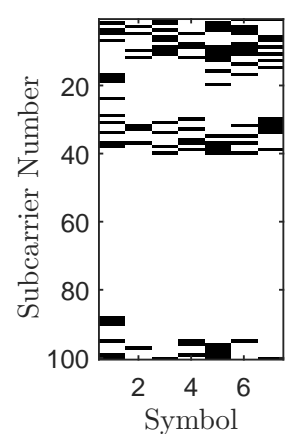

(a) Open (Prime)

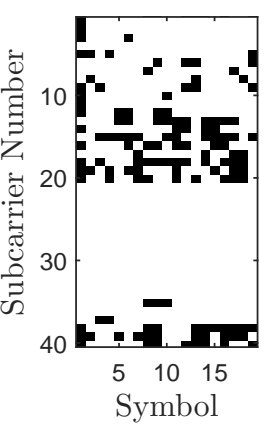

(d) Open (G3-PLC)

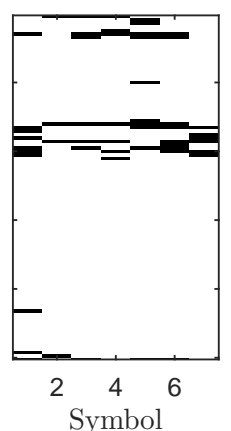

(b) Mixed (Prime)

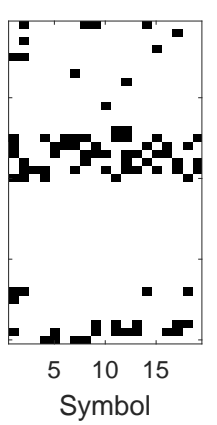

(e) Mixed (G3PLC)

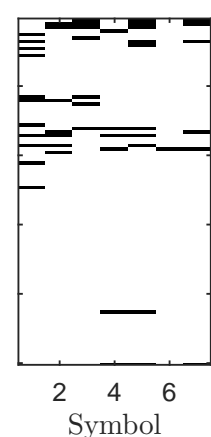

(c) Sabiha (Prime)
Fig. 12. Diagrammatic representations of bit errors mapped against subcarrier for Prime communication between nodes D and B and for Open, Mixed and Sabiha transformer terminations. The black lines represent errors, and are concentrated in bands, demonstrating frequency selectivity.

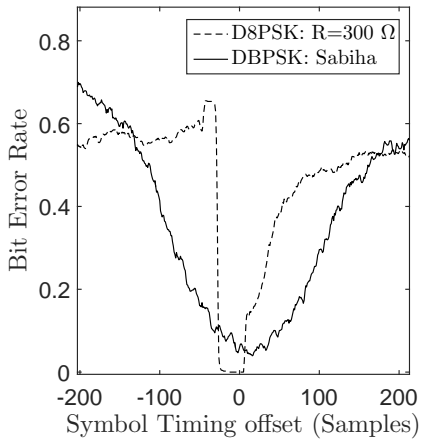

(a) Prime

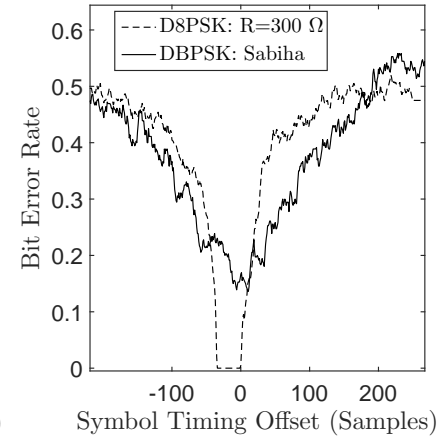

(b) G3-PLC
Fig. 13. BER as a function of symbol timing offset for $\mathrm{R}=300 \Omega$ and Sabiha transformer terminations.

\section{E. Robustness to Symbol Timing Offset}

In differential OFDM schemes, there is a BER penalty due timing misalignment. When the timing offset falls back to within the $\mathrm{CP}$, each subcarrier will be shifted in frequency by a differing amount (the higher the frequency, the greater the phase shift). This presents itself as an impairment, with the phase error given by the following equation:

$$
\Phi_{e}=w \frac{2 \pi}{k}
$$

Where $w$ is the number of symbol timing offsets and $k$ is the number of subcarriers. Prime, which encodes data in 


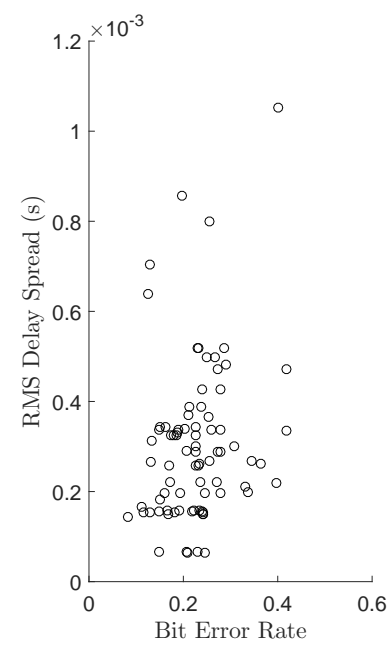

(a) DQPSK Prime

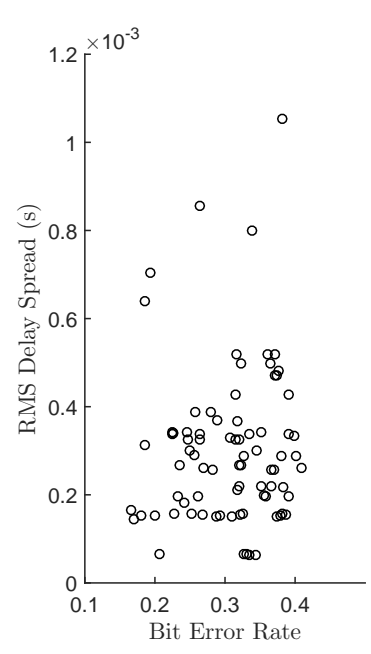

(b) D8PSK Prime

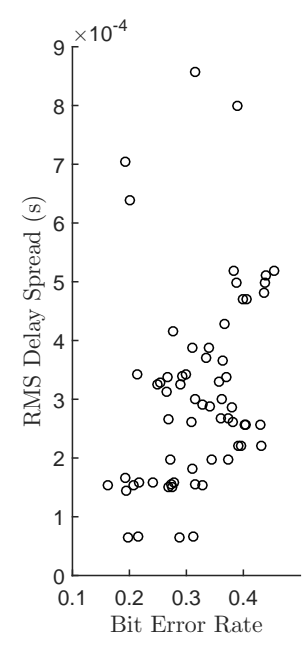

(c) DQPSK G3PLC

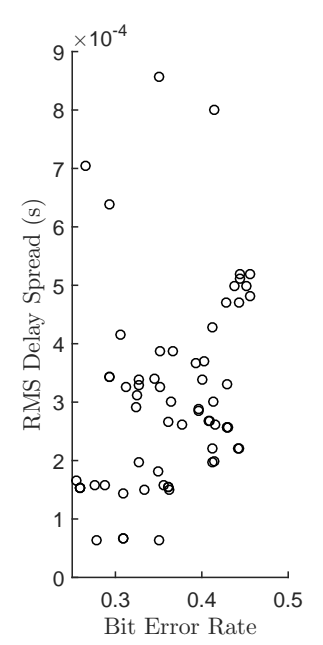

(d) D8PSK G3PLC

Fig. 14. Scatter plots of BER versus RMS Delay spread for all possible channels (between nodes A to E) on the test network.

frequency, is expected to be more prone to this error than G3-PLC, which encodes in time. Fig. 13 shows the BER performance of Prime and G3-PLC for a range of symbol timing offsets, i.e. misalignments between the receiver FFT sampling window and the symbol to be demodulated. In the $\mathrm{R}=300 \Omega$ termination case, which may be regarded as a good quality channel with little multipath, the BER plateaus to zero for small negative timing offsets (i.e. where the FFT window begins early, and is therefore within the $\mathrm{CP}$ ). For Prime, there is a cliff-edge effect increase in BER at around 20-25 samples due to the cyclically shifted symbols rotating, en masse, across the decision threshold. In G3-PLC, this effect is less noticeable because the cyclic shifting caused by the misaligned window is the same in consecutive symbols. In a channel severely affected by multipath (chosen here as the Sabiha terminated case), both Prime and G3-PLC have a "U" shape, with a degree of symmetry between the penalties associated with negative and positive offsets.

\section{F. Relationship between RMS Delay Spread and BER}

Fig. 14 shows scatter plots of BER versus RMS delay spread for every possible channel involving nodes $A$ to $\mathrm{E}$ and for all termination scenarios. For all cases, there is an observable correlation between delay spread and BER. This result demonstrates that the BER is somewhat sensitive to the delay spread of the particular channel; an expected result given the evidence from work carried out on the mobile channel, where a proportionate relationship between the irreducible error floor and the RMS-DS normalised to the bit period has been established [22]. For G3-PLC, this would predict a relatively high irreducible error floor because the symbol duration is large compared to the typical RMS-DS observed in the test network. Given that the RMS-DS is a property of the channel itself, it is recommended that both the CP and symbol duration are increased to accommodate for this. However, there is little information in the literature on the empirical measurement of the RMS delay spread on the narrowband
MV power line channel. For PLC to operate robustly on MV networks in the narrowband range, it is crucial that the standards properly set their CP and symbol durations for this type of channel.

\section{CONCLUSION}

The EMTP is a well trusted tool for frequency dependent transient studies, though has not yet been exploited to its full potential for PLC studies. In this work, the EMTPATP has been used to assess, both the channel properties of the MV channel and the subsequent performance of the emerging Prime and G3-PLC standards. It has been shown that the RMS delay spread and coherence bandwidth is highly dependent on how many branches are terminated by a transformer, with the open circuit assumption exhibiting the largest RMS delay spread, the most severe frequency selective fading and the highest irreducible error floor. The performance of Prime and G3-PLC is exceptionally good when all branches are terminated by a resistor approximating the characteristic impedance of the line, but relatively poor when all branches are open circuited or terminated by a transformer model. The simulations also show an improvement in BER performance as the $\mathrm{CP}$ is increased. They also show a considerable increase in performance when the sampling frequency is halved, indicating that the severity of the channel may not properly be accounted for in the Prime and G3-PLC standards. In particular, the $\mathrm{CP}$ and symbol duration is set too short in G3-PLC, and the subcarrier spacing set too large in Prime for optimal performance. Given that bit errors are localised, adaptive schemes have an important role to play in these standards, and there is good promise for these technologies if they can successfully navigate away from the regions severely affected by multipath interference. Future work will attempt to compare the results of the EMTP simulations with actual measurement. 


\section{REFERENCES}

[1] Y. Yan, Y. Qian, H. Sharif, and D. Tipper, "A survey on smart grid communication infrastructures: Motivations, requirements and challenges," Communications Surveys Tutorials, IEEE, vol. 15, no. 1, pp. 5-20, 2013.

[2] A. Milioudis, G. Andreou, and D. Labridis, "Enhanced protection scheme for smart grids using power line communications techniques 2014;part i: Detection of high impedance fault occurrence," Smart Grid, IEEE Transactions on, vol. 3, no. 4, pp. 1621 -1630, dec. 2012.

[3] B. Adebisi, A. Treytl, A. Haidine, A. Portnoy, R. Shan, D. Lund, H. Pille, and B. Honary, "Ip-centric high rate narrowband plc for smart grid applications," Communications Magazine, IEEE, vol. 49, no. 12, pp. $46-54$, december 2011.

[4] V. Levi, G. Strbac, and R. Allan, "Assessment of performance-driven investment strategies of distribution systems using reference networks," Generation, Transmission and Distribution, IEE Proceedings-, vol. 152, no. 1 , pp. $1-10$, jan. 2005 .

[5] Narrowband orthogonal frequency division multiplexing power line communication transceivers for PRIME networks, ITU-T Std. G.9904, 2013.

[6] Narrowband orthogonal frequency division multiplexing power line communication transceivers for G3-PLC networks, ITU-T Std. G.9903, 2014.

[7] Signalling on low-voltage electrical installations in the frequency range $3 \mathrm{kHz}$ to $148.5 \mathrm{kHz}$, CENELEC Std. EN 50 065-1, 1991.

[8] G. Artale, A. Cataliotti, V. Cosentino, D. Di Cara, R. Fiorelli, P. Russotto, and G. Tine, "Medium voltage smart grid: Experimental analysis of secondary substation narrow band power line communication," Instrumentation and Measurement, IEEE Transactions on, vol. 62, no. 9, pp. 2391-2398, Sept 2013.

[9] G. Ault, "United kingdom generic distribution system (ukgds)," DTI Centre for Distributed Generation and Sustainable Electrical Energy, 2010.

[10] J. Marti, "Accurate modelling of frequency-dependent transmission lines in electromagnetic transient simulations," Power Apparatus and Systems, IEEE Transactions on, vol. PAS-101, no. 1, pp. 147 -157, jan. 1982.

[11] S. Carneiro and J. R. Marti, "Evaluation of corona and line models in electromagnetic transients simulations," IEEE Transactions on Power Delivery, vol. 6, no. 1, pp. 334-342, Jan 1991.

[12] T. Tran-Anh, P. Auriol, and T. Tran-Quoc, "High frequency power transformer modeling for power line communication applications," in Power Systems Conference and Exposition, 2006. PSCE '06. 2006 IEEE PES, Oct 2006, pp. 1069-1074.

[13] N. A. Sabiha and M. Lehtonen, "Lightning-induced overvoltages transmitted over distribution transformer with mv spark-gap operation x2014;part i: High-frequency transformer model," IEEE Transactions on Power Delivery, vol. 25, no. 4, pp. 2472-2480, Oct 2010.

[14] A. Cataliotti, V. Cosentino, D. D. Cara, and G. Tinè, "Oil-filled mv/lv power-transformer behavior in narrow-band power-line communication systems," Instrumentation and Measurement, IEEE Transactions on, vol. 61, no. 10, pp. 2642-2652, 2012.

[15] A. G. Lazaropoulos and P. G. Cottis, "Transmission characteristics of overhead medium-voltage power-line communication channels," IEEE Transactions on Power Delivery, vol. 24, no. 3, pp. 1164-1173, July 2009.

[16] M. Tlich, A. Zeddam, F. Moulin, and F. Gauthier, "Indoor power-line communications channel characterization up to $100 \mathrm{mhz}$ x2014;part ii: Time-frequency analysis," Power Delivery, IEEE Transactions on, vol. 23, no. 3, pp. $1402-1409$, july 2008 .

[17] A. Cataliotti, D. Di Cara, R. Fiorelli, and G. Tine, "Power-line communication in medium-voltage system: Simulation model and onfield experimental tests," Power Delivery, IEEE Transactions on, vol. 27, no. 1 , pp. $62-69$, jan. 2012.

[18] S. Barmada, A. Musolino, and M. Raugi, "Innovative model for timevarying power line communication channel response evaluation," Selected Areas in Communications, IEEE Journal on, vol. 24, no. 7, pp. 1317 - 1326, july 2006.

[19] J. Anatory, N. Theethayi, and R. Thottappillil, "Power-line communication channel model for interconnected networks x2014; part ii: Multiconductor system," Power Delivery, IEEE Transactions on, vol. 24, no. 1, pp. $124-128$, jan. 2009.

[20] M. Zimmermann and K. Dostert, "A multipath model for the powerline channel," Communications, IEEE Transactions on, vol. 50, no. 4, pp. $553-559$, apr 2002

[21] L. Hanzó, W. Webb, and T. Keller, Single- and multi-carrier quadrature amplitude modulation: principles and applications for personal coтmu- nications, WLANs and broadcasting. John Wiley \& Sons, 2000. [Online]. Available: http://books.google.co.uk/books?id=sO9SAAAAMAAJ

[22] J. Chuang, "The effects of time delay spread on portable radio communications channels with digital modulation," IEEE Journal on Selected Areas in Communications, vol. 5, no. 5, pp. 879-889, Jun 1987.

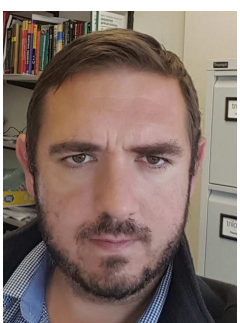

Stephen Robson (M14) received the M.Eng. degree in 2007 and the Ph.D. degree in 2012. Between 2007 and 2008, he worked as a graduate engineer with National Grid UK. In 2013, he was appointed as a Lecturer in Electrical Engineering with Cardiff University. His main research interests are in power line communication, fault location, condition monitoring, and simulation of transients on electrical networks. $\mathrm{He}$ is a member of the IEEE.

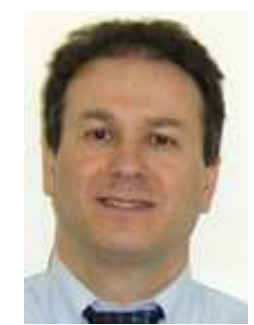

Manu Haddad (M'13) received the degree of of Ingnieur d'tat in electrical engineering in 1985 and the Ph.D. degree in high-voltage engineering in 1990. He is currently a Professor of Electrical Engineering with Cardiff University responsible for research in high-voltage engineering. He has authored an IET- Power Series Book entitled Advances in High-Voltage Engineering. His research interests are in overvoltage protection, insulation systems, insulation coordination, and earthing of electrical energy systems. He is a member of several CIGRE Working Groups and a member of the BSI PEL1/2, the IEC TC37 and the IEC ACTAD committees MT4 and MT10. He serves on the scientific committees of several international conferences. He is a fellow of the IET and the Learned Society of Wales

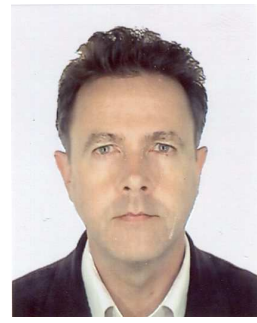

Huw Griffiths (M'13) received the B.Sc. degree in 1982, and received the Ph.D. degree from Cardiff University, U.K. From 1983 to 1990 , he was with the South Wales Electricity Board and the Central Electricity Generating Board, where he was an 723 Engineer in distribution and transmission system design. In 1990, he was appointed as a Lecturing Staff Member with Cardiff University. Since 2015, he has been a Professor of Power systems and HighVoltage Engineering with the Petroleum Institute, Abu Dhabi, prior to that with Cardiff University. His research interests include earthing systems and transients. He is currently a member of British Standard Institution Committees BSI PEL/99 (HV ac substations), the Chair of BSI GEL/600 (earthing), and a member of CENELEC TC99X WG1, Earthing of Power Installations exceeding 1-kV ac, and the IEC Committee TC99/MT4-IEC 61936 Power Installations exceeding $1-\mathrm{kV}$ ac. He is a Chartered Engineer and a member of the IET. 